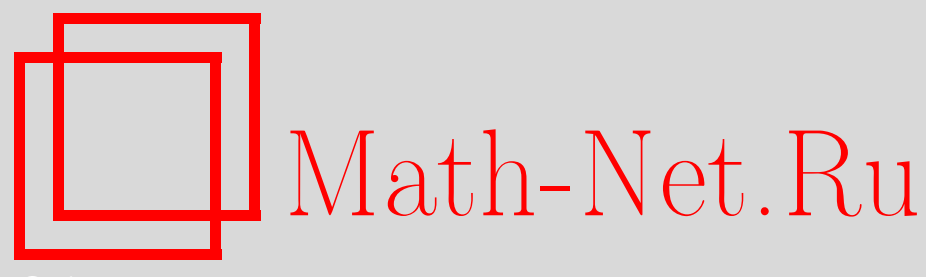

Ян Ли, Юань Сун, Появление гигантской компоненты в растущих сетях с преимущественным прирастанием, TMФ, 2016, том 186, номер 3, 496-507

DOI: https://doi.org/10.4213/tmf8939

Использование Общероссийского математического портала Math-Net.Ru подразумевает, что вы прочитали и согласны с пользовательским соглашением http://www . mathnet.ru/rus/agreement

Параметры загрузки:

IP: 52.23 .180 .231

26 апреля 2023 г., 18:18:43

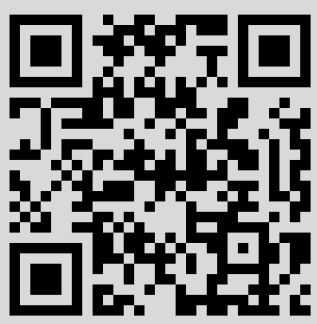




\title{
ПОЯВЛЕНИЕ ГИГАНТСКОЙ КОМПОНЕНТЫ В РАСТУШИХ СЕТЯХ С ПРЕИМУЩЕСТВЕННЫМ ПРИРАСТАНИЕМ
}

\begin{abstract}
Исследуется перколяционный переход в эволюционирующих безмасштабных сетях. На каждом шаге эволюции добавляется новый узел, который связывается со случайным числом предыдущих узлов в соответствии с механизмом преимущественного прирастания. Определено критическое значение параметра модели, при котором в сети возникает гигантская компонента, и доказано, что переход имеет бесконечный порядок. Кроме того, получены асимптотические выражения для распределения кластеров по их размерам в подкритическом, критическом и надкритическом режимах.
\end{abstract}

Ключевые слова: безмасштабные сети, перколяция, переход Костерлица-Зоулесса, распределение кластеров по размерам.

DOI: $10.4213 / \operatorname{tmf} 8939$

\section{1. ВВЕДЕНИЕ}

Многие реально существующие сети, такие как интернет, энергетические сети, социальные сети и т. д., подчиняются безмасштабному распределению по степеням [1], согласно которому доля $p_{k}$ узлов, имеющих степень (число инцидентных связей) $k$, асимптотически равна $C k^{-\alpha}$, где $C>0$ и $\alpha>1$. Это наблюдение и практическая важность таких сетей мотивировали интенсивные исследования структурных свойств безмасштабных сетей [2], [3].

Возникновение безмасштабных сетей часто объясняется преимущественным прирастанием. В знаменитой модели Барабаши-Альберта (БА) [4] на каждом шаге алгоритма добавляется один узел, связанный с $m$ существующими узлами, а вероятность $\Pi_{i}$ получения новой связи для узлов $i$ пропорциональна степени $d_{i}$ этого узла, т. е. $\Pi_{i}=d_{i} / \sum_{i} d_{i}$. Были предложены различные обобщения модели БА, включающие другие типы преимущественного прирастания вероятности $\Pi_{i}[5]$, нелинейные

Работа поддержана National Natural Science Foundation of China (грант № 11401368).

* School of Mathematics and Physics, Shanghai University of Electric Power, Shanghai, China. E-mail: liyan@shiep.edu.cn 
степени роста [6], введение весов на связях [7], динамическое перераспределение связей [8] и т. д. Были достаточно хорошо исследованы многие аспекты этих моделей, например распределение степеней и коэффициент кластеризации [9].

Среди критических явлений в подобных сетях особый интерес вызывает перколяционное поведение, поскольку с его помощью измеряется связность и устойчивость сети [10]-[13]. В хорошо изученной модели случайных графов, введенной Эрдошем и Реньи (ЭР), одним из наиболее интересных явлений оказывается существование порогового значения параметра модели, при котором появляется гигантская компонента связности [14], [15]. Естественно задать вопрос, существуют ли такие перколяционные переходы в динамически растущих сетях? В этой связи в работах [16] и [17] было показано, что перколяционный переход в простых растущих сетях проявляет свойства, весьма отличающиеся от свойств такого перехода в модели ЭР.

В настоящей работе предлагается модель растущей сети, в которой на каждом шаге роста в соответствии с правилом преимущественного прирастания к сети добавляется один новый узел со случайным числом связей, связывающих этот узел с существующими ранее. В отличие от модели БА, которая содержит только одну компоненту связности (кластер), в данной сети возможно существование нескольких кластеров, так как добавляемые новые узлы могут с ненулевой вероятностью оказаться изолированными. Детально исследуется явление перколяции в модели сети с таким преимущественным прирастанием. Доказано, что в модели имеется перколяционный переход бесконечного порядка. Получено выражение для размера гигантского кластера и асимптотическое распределение кластеров по размерам в подкритическом, околокритическом и критическом режимах. Авторы надеются, что эта сравнительно простая модель позволит прояснить некоторые универсальные свойства перколяции в эволюционирующих безмасштабных сетях.

Рассмотренная нами модель была инспирирована работой Дороговцева с соавторами [17], в которой на каждом шаге эволюции в модель вводился новый узел и в соответствии с правилом преимущественного прирастания добавлялись новые связи между узлами. Основное отличие нашей модели состоит в том, что в ней новые связи добавляются только между новым узлом и старыми узлами, а в модели из работы [17] новые связи добавляются между любыми двумя узлами сети. Несмотря на это отличие, обе модели проявляют совпадающие перколяционные свойства такие, как бесконечный порядок перколяционного перехода и асимптотика распределения кластеров по размерам. Это можно объяснить тем, что в работе [17] два кластера соединяются напрямую новыми добавляемыми связями, а в нашей модели они соединяются опосредованным образом путями, проходящими через новый узел. Поэтому механизм роста кластеров в обеих моделях оказывается весьма схожим.

Работа организована следующим образом. Модель и основные результаты представлены в разделе 2. Доказательства всех теорем даются в разделе 3. Наконец, в разделе 4 обсуждается обобщение данной модели.

Следует отметить, что, хотя модель, рассматриваемая в настоящей работе, отличается от модели, предложенной Каллавеем, Хопкрофтом, Клейнбергом, Ньюманом и Строгацем (KXKHC) [16], равно как и от ее обобщений [17]-[20], упомянутые работы обогатили авторов многочисленными ценными идеями и методами вычислений. 


\section{2. МОДЕЛЬ И ОСНОВНЫЕ РЕЗУЛЬТАТЫ}

Барабаши и Альберт указали, что разрастание и преимущественное прирастание оказываются двумя определяющими свойствами, которые приводят к появлению безмасштабных сетей [4], поэтому эти два механизма содержатся и в нашей модели. Вместо добавления фиксированного числа связей на каждом этапе предполагается, что число новых связей представляет собой случайную величину с распределением $\boldsymbol{\tau}=\left\{\tau_{k}, k \geqslant 0\right\}$. Более точно, на каждом этапе добавляется новый узел, из которого с вероятностью $\tau_{k}$ выходят $k$ связей, соединяющих ее со старыми узлами. Вероятность того, что узел $i$ будет при этом связанным с новым узлом, равна

$$
\Pi\left(d_{i}\right)=\frac{d_{i}+a}{\sum_{j}\left(d_{j}+a\right)},
$$

где $a>0$ - исходная привлекательность, позволяющая изолированным узлам приобретать новые связи. Чтобы работать с моделью было легче, предположим, что $\boldsymbol{\tau}$ - распределение Пуассона с параметром $\lambda$,

$$
\tau_{k}=\frac{\lambda^{k}}{k !} e^{-\lambda}, \quad k=0,1,2, \ldots .
$$

В разделе 4 обсуждаются другие, более общие, распределения.

Оказывается, что вид распределения $\boldsymbol{\tau}$ не слишком сильно влияет на распределение $\left\{p_{k}\right\}$ степеней узлов, и получающаяся при этом сеть по-прежнему оказывается безмасштабной. Можно доказать, что $p_{k} \sim C k^{-\alpha}$ с $\alpha=3+a / \lambda$ при $k \rightarrow \infty$. Заметим, что $\alpha \rightarrow 3$ при $a \rightarrow 0$, и это находится в согласии с показателем степени модели БА [4].

Наша модель отличается от модели БА тем, что новый узел при добавлении его к сети не обязательно связан со старыми узлами, так как $\tau_{0}=e^{-\lambda}>0$. Вследствие этого сеть в общем случае оказывается несвязной, и в ней возникают кластеры различных размеров. Основная цель настоящей работы - исследование фазового перехода к появлению гигантского кластера. Первый результат состоит в определении критического значения $\lambda$, при котором происходит этот переход.

ОПРЕДЕЛЕНИЕ 1 . Пусть $N_{k}(t)$ - математическое ожидание числа компонент связности (кластеров) размера $k$ в момент времени $t$, а $n_{k}=\lim _{t \rightarrow \infty} N_{k}(t) / t$ - соответствующая предельная плотность. Обозначим через

$$
S=1-\sum_{k=1}^{\infty} k n_{k}
$$

плотность кластера бесконечного размера. Если существует значение $\lambda_{\mathrm{c}}$ такое, что $S=0$ при $\lambda \leqslant \lambda_{\text {с и }} S>0$ при $\lambda>\lambda_{\text {c }}$, то будем называть $\lambda_{\mathrm{c}}$ критическим значением для возникновения гигантской компоненты.

Tеорема 1. При каждом $a \geqslant 0$

$$
\lambda_{\mathrm{c}}=\frac{a}{2}\left(a+1-\sqrt{a^{2}+2 a}\right) .
$$




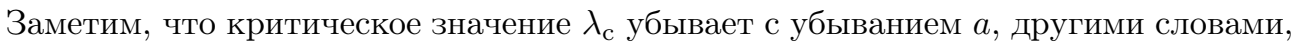
оно убывает с усилением тенденции преимущественного разрастания. В предельном случае $a=0$ всегда имеется кластер бесконечного размера $\left(\lambda_{\mathrm{c}}=0\right)$, так как изолированные узлы никогда не обзаводятся новыми связями. При $a \rightarrow \infty$ преимущественное присоединение не имеет места, и мы получаем $\lambda_{\mathrm{c}} \rightarrow 1 / 4$, что совпадает с ответом из работы [18].

Чтобы изучить характер фазового перехода, приведем оценку среднего размера кластера и предельной плотности бесконечной компоненты в окрестности точки $\lambda_{c}$.

Теорема 2. Обозначим через $\langle s\rangle=\sum_{k=1}^{\infty} k^{2} n_{k}$ среднее значение конечной компоненты. Имеем

$$
\begin{array}{ll}
\langle s\rangle \rightarrow \frac{4 \lambda_{\mathrm{c}}^{2}-2 a^{2} \lambda_{\mathrm{c}}+a^{2}}{2(2+a)^{2} \lambda_{\mathrm{c}}^{2}}, & \lambda \uparrow \lambda_{\mathrm{c}}, \\
\langle s\rangle \rightarrow \frac{4(1-a) \lambda_{\mathrm{c}}^{2}+a(2-a) \lambda_{\mathrm{c}}+a^{2}}{(2+a)^{2} \lambda_{\mathrm{c}}^{2}}, & \lambda \downarrow \lambda_{\mathrm{c}} .
\end{array}
$$

TEOPEMA 3. При $\lambda \downarrow \lambda_{\mathrm{c}}$

$$
S=C \frac{(2 \lambda+a)^{2}}{\lambda^{2}(2+a)} \exp \left(-\frac{\pi}{\sqrt{4 \kappa-1}}\right), \quad C>0, \quad \kappa=\frac{a(2+a) \lambda}{(2 \lambda+a)^{2}} .
$$

Заметим, что в результате появления гигантской компоненты величина $\langle s\rangle$ ста-

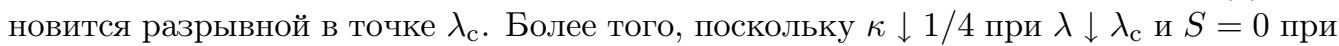
$\lambda \leqslant \lambda_{\mathrm{c}}$, с помощью соотношения (1) можно проверить, что производные функции $S$ любого порядка обращаются в нуль в точке $\lambda_{c}$. Поэтому перколяционный переход имеет бесконечный порядок (переход типа Костерлица-Зоулесса), что также наблюдалось в модели КХKНС [16]-[18], [20].

Наконец, рассмотрим распределение $q_{k}=k n_{k}$ кластеров по их размерам. При фиксированном $k$ величина $q_{k}$ интерпретируется как значение правдоподобия при случайном процессе выбора узла, принадлежащего кластеру размера $k$. Приведенная ниже теорема дает асимптотические выражения для $q_{k}$ в подкритической, околокритической и надкритической областях.

ТЕОРема 4. При $k \rightarrow \infty$ имеют место следующие асимптотики.

1. $\operatorname{Ec} л u \lambda=\lambda_{\mathrm{c}}$, mo

$$
q_{k} \approx \frac{\left(2 \lambda_{\mathrm{c}}+a\right)^{2}}{2 \lambda_{\mathrm{c}}^{2}(2+a)^{2}} \frac{1}{k^{2} \ln ^{2} k}
$$

2. $Е с л и \lambda<\lambda_{\mathrm{c}}$, mo

$$
q_{k} \approx C_{1} \frac{1}{k^{2+\Lambda}}, \quad C_{1}=\mathrm{const}, \quad \Lambda=\frac{4 \omega}{1-2 \omega}, \quad \omega=\sqrt{\frac{1}{4}-\kappa}, \quad \kappa=\frac{a(2+a) \lambda}{(2 \lambda+a)^{2}} .
$$

3. Если $\lambda>\lambda_{\mathrm{c}}$, mo

$$
q_{k} \approx C_{2} k^{-1 / 2} \exp \left[-C_{3} e^{-\pi / 2 \eta-1} k-\frac{3 \pi}{4 \eta}-\frac{3}{2}\right], \quad C_{2}, C_{3}=\text { const, } \quad \eta=\sqrt{\kappa-\frac{1}{4}} .
$$

Из теоремы 4 следует, что распределение кластеров по размерам имеет степенную зависимость до возникновения гигантского кластера и становится экспоненциальной в надкритической области. 


\section{3. ДОКАЗАТЕЛЬСТВА ТЕОРЕМ}

ДоКАЗАТЕЛЬСТво ТЕОРЕмЫ 1. Ключевым моментом при выводе уравнений роста для $n_{k}$ оказывается то, что почти наверное все кластеры конечного размера являются древесными графами [14], [15], [17]. Поэтому кластер размера $k$ содержит $k-1$ связей, и его полная степень равна $2(k-1)$. В совокупности с тем обстоятельством, что математическое ожидание полного числа связей в момент времени $t$ равно $\lambda t$, вероятность того, что возникла новая связь с кластером, равна $[(2+a) k-2] /[(2 \lambda+a) t]$. Поэтому величина $N_{k}(t)$ удовлетворяет уравнению

$$
\begin{aligned}
N_{k}(t+1)-N_{k}(t)= & -\lambda \frac{(2+a) k-2}{(2 \lambda+a) t} N_{k}(t)+ \\
& +\sum_{n=0}^{\infty} \frac{\lambda^{n}}{n !} e^{-\lambda} \sum_{k_{1}+\cdots+k_{n}=k-1} \prod_{j=1}^{n} \frac{(2+a) k_{j}-2}{(2 \lambda+a) t} N_{k_{j}}(t),
\end{aligned}
$$

в котором первый член в правой части описывает убывание величины $N_{k}$, обусловленное связью между новым узлом и кластерами размера $k$, а второй член описывает слияние кластеров в кластер размера $k$.

В предельном случае $t \rightarrow \infty$ из уравнения (2) получаем

$$
n_{k}=-\lambda \frac{(2+a) k-2}{2 \lambda+a} n_{k}+\sum_{n=0}^{\infty} \frac{\lambda^{n}}{n !} e^{-\lambda} \sum_{k_{1}+\cdots+k_{n}=k-1} \prod_{j=1}^{n} \frac{(2+a) k_{j}-2}{2 \lambda+a} n_{k_{j}} .
$$

Вводя производящие функции

$$
f(z)=\sum_{k=1}^{\infty} n_{k} e^{k z}, \quad g(z)=f^{\prime}(z)=\sum_{k=1}^{\infty} k n_{k} e^{k z},
$$

приведем уравнение (3) к виду

$$
g^{\prime}(z)=\frac{H_{1}(z)}{H_{2}(z)},
$$

где

$$
\begin{aligned}
& H_{1}(z)=-\frac{a}{2 \lambda+a} g(z)+\left[1-\frac{2 \lambda}{2 \lambda+a} g(z)\right] \exp \left[-\lambda+z+\frac{\lambda(2+a)}{2 \lambda+a} g(z)-\frac{2 \lambda}{2 \lambda+a} f(z)\right], \\
& H_{2}(z)=\frac{\lambda(2+a)}{2 \lambda+a}-\frac{\lambda(2+a)}{2 \lambda+a} \exp \left[-\lambda+z+\frac{\lambda(2+a)}{2 \lambda+a} g(z)-\frac{2 \lambda}{2 \lambda+a} f(z)\right] .
\end{aligned}
$$

Так как на каждом этапе добавляется один узел и $\lambda$ связей, получим

$$
f(0)=\sum_{k} n_{k}=1-\lambda .
$$

Если гигантский кластер отсутствует, выполняется соотношение $g(0)=f^{\prime}(0)=1$, и можно показать, что $H_{1}(0)=H_{2}(0)=0$. Из правила Лопиталя следует, что

$$
\langle s\rangle=g^{\prime}(0)=\lim _{z \rightarrow 0} \frac{H_{1}^{\prime}(z)}{H_{2}^{\prime}(z)}=\frac{-g^{\prime}(0)+\frac{a}{2 \lambda+a}\left[\frac{a}{2 \lambda+a}+\frac{\lambda(2+a)}{2 \lambda+a} g^{\prime}(0)\right]}{-\frac{\lambda(2+a)}{2 \lambda+a}\left[\frac{a}{2 \lambda+a}+\frac{\lambda(2+a)}{2 \lambda+a} g^{\prime}(0)\right]} .
$$


Переставляя члены, получим

$$
\lambda^{2}(2+a)^{2}\left[g^{\prime}(0)\right]^{2}+\left(2 \lambda a^{2}-a^{2}-4 \lambda^{2}\right) g^{\prime}(0)+a^{2}=0 .
$$

Решение этого квадратного уравнения имеет вид

$$
g^{\prime}(0)=\frac{-\left(2 \lambda a^{2}-a^{2}-4 \lambda^{2}\right) \pm \sqrt{\left(2 \lambda a^{2}-a^{2}-4 \lambda^{2}\right)^{2}-4 \lambda^{2}(2+a)^{2} a^{2}}}{2 \lambda^{2}(2+a)^{2}} .
$$

Поскольку $\langle s\rangle=g^{\prime}(0) \rightarrow 1$ при $\lambda \rightarrow 0$, решение с плюсом перед радикалом следует отбросить.

Фазовый переход возникает при

$$
\left(2 \lambda a^{2}-a^{2}-4 \lambda^{2}\right)^{2}-4 \lambda^{2}(2+a)^{2} a^{2}=0,
$$

и получающееся при этом физическое решение имеет вид

$$
\lambda_{\mathrm{c}}=\frac{a}{2}\left(a+1-\sqrt{a^{2}+2 a}\right) .
$$

Теорема доказана.

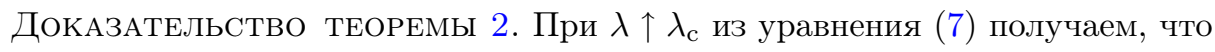

$$
\langle s\rangle=g^{\prime}(0) \rightarrow \frac{4 \lambda_{\mathrm{c}}^{2}-2 a^{2} \lambda_{\mathrm{c}}+a^{2}}{2(2+a)^{2} \lambda_{\mathrm{c}}^{2}} .
$$

Если $\lambda>\lambda_{\mathrm{c}}$, то $g(0)<1$ и гигантский кластер имеет размер

$$
S=1-g(0)
$$

Подстановка $z=0$ в равенство (5) дает

$$
g^{\prime}(0)=\frac{-\frac{a}{2 \lambda+a}(1-S)+\left[1-\frac{2 \lambda}{2 \lambda+a}(1-S)\right] \exp \left[-\lambda+\frac{\lambda(2+a)}{2 \lambda+a}(1-S)-\frac{2 \lambda}{2 \lambda+a}(1-\lambda)\right]}{\frac{\lambda(2+a)}{2 \lambda+a}-\frac{\lambda(2+a)}{2 \lambda+a} \exp \left[-\lambda+\frac{\lambda(2+a)}{2 \lambda+a}(1-S)-\frac{2 \lambda}{2 \lambda+a}(1-\lambda)\right]} .
$$

Так как $S \rightarrow 0$ при $\lambda \downarrow \lambda_{\mathrm{c}}$, мы получаем

$$
\langle s\rangle=g^{\prime}(0) \rightarrow \frac{4(1-a) \lambda_{\mathrm{c}}^{2}+a(2-a) \lambda_{\mathrm{c}}+a^{2}}{(2+a)^{2} \lambda_{\mathrm{c}}^{2}} .
$$

Теорема доказана.

ДОКАЗАТЕЛЬСТВО ТЕОРЕМЫ 3. Пусть

$$
h(z)=-\lambda+z+\frac{\lambda(2+a)}{2 \lambda+a} g(z)-\frac{2 \lambda}{2 \lambda+a} f(z),
$$

где $f(z)$ и $g(z)$ заданы в (4). Преобразуя выражение (5), получаем

$$
g(z)=1-h^{\prime}(z)+e^{h(z)} h^{\prime}(z) .
$$


При $z \rightarrow-\infty$ мы имеем $f(z) \rightarrow 0, g(z) \rightarrow 0, h(z) \rightarrow-\infty$ и $z-h(z) \rightarrow \lambda$, таким образом,

$$
f(z)=\int_{-\infty}^{z} g(t) d t=\int_{-\infty}^{z}\left[1-h^{\prime}(z)+e^{h(z)} h^{\prime}(z)\right] d t=z-h(z)+e^{h(z)}-\lambda .
$$

Подстановка соотношений (11) и (12) в (10) дает

$$
h(z)=\frac{a}{2 \lambda+a} z+\frac{\lambda(2+a)}{2 \lambda+a}\left[e^{h(z)}-1\right] h^{\prime}(z)-\frac{2 \lambda}{2 \lambda+a}\left[e^{h(z)}-h(z)-1\right] .
$$

Используя разложение $e^{h(z)}$ в окрестности точки $z=0$,

$$
e^{h(z)}=1+h(z)+\frac{1}{2} h^{2}(z)+\cdots,
$$

получаем, что уравнение (13) можно аппроксимировать дифференциальным уравнением Абеля второго рода

$$
h(z) h^{\prime}(z)=\frac{1}{2+a} h^{2}(z)-\frac{2 \lambda+a}{\lambda(2+a)} h(z)-\frac{a}{\lambda(2+a)} z .
$$

С помощью подстановок

$$
u=e^{-z /(2+a)}, \quad w(u)=\frac{\lambda}{2 \lambda+a} e^{-z /(2+a)} h(z)
$$

приведем уравнение (14) к более простому виду:

$$
w(u) w^{\prime}(u)-w(u)=-\kappa u \ln u, \quad \text { где } \quad \kappa=\frac{a(2+a) \lambda}{(2 \lambda+a)^{2}} .
$$

В окрестности $z=0$ имеем $u \approx 1$ и $\ln u \approx u-1$, поэтому уравнение (16) можно записать как

$$
w(u) w^{\prime}(u)-w(u)=-\kappa(u-1) .
$$

Решение этого уравнения имеет вид [21]

$$
\ln (u-1)+\int \frac{\tau d \tau}{\tau^{2}-\tau+\kappa}=C,
$$

где $\tau=w(u) /(u-1)$.

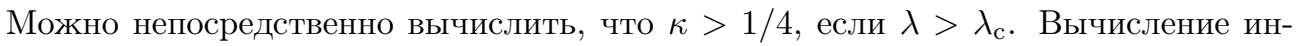
теграла в выражении (18) дает

$$
\ln \sqrt{w^{2}(u)-w(u)(u-1)+\kappa(u-1)^{2}}-\frac{1}{\sqrt{4 \kappa-1}} \operatorname{arctg} \frac{1-2 w(u) /(u-1)}{\sqrt{4 \kappa-1}}=C .
$$

Положив $u=1$, с учетом соотношений $(6),(9),(10)$ и (15) получаем, что

$$
C=\ln \frac{\lambda^{2}(2+a)}{(2 \lambda+a)^{2}} S+\frac{1}{\sqrt{4 \kappa-1}} \frac{\pi}{2} .
$$


При $\lambda \downarrow \lambda_{\mathrm{c}}$ (при $\left.\kappa \downarrow 1 / 4\right)$ с использованием асимптотического выражения

$$
\operatorname{arctg} x=\frac{\pi}{2}-\operatorname{arctg} \frac{1}{x} \approx \frac{\pi}{2}-\frac{1}{x}, \quad x \rightarrow+\infty,
$$

равенство (19) приводится к виду

$$
\ln \left[\frac{u-1}{2}-w(u)\right]-\frac{\pi}{\sqrt{4 \kappa-1}}+\frac{1}{1-2 w(u) /(u-1)}=\ln \frac{\lambda^{2}(2+a)}{(2 \lambda+a)^{2}} S .
$$

В точке $\lambda=\lambda_{\text {c }}$ (при $\left.\kappa=1 / 4\right)$ после подстановки

$$
w(u)=\frac{1}{2}(u-1)[1-\varphi(u)]
$$

решение уравнения (17) задается выражением

$$
\ln [(u-1) \varphi(u)]+\frac{1}{\varphi(u)}=\ln C_{1}
$$

Комбинируя соотношения (21)-(23), в конечном итоге получаем

$$
S=C_{1} \frac{(2 \lambda+a)^{2}}{\lambda^{2}(2+a)} \exp \left[-\frac{\pi}{\sqrt{4 \kappa-1}}\right] .
$$

Теорема доказана.

ДоКАЗАТЕЛЬСТво тЕОРЕмы 4. Обратное Z-преобразование функции $g(z)$ дает

$$
q_{k}=\frac{1}{2 \pi i} \int_{-\pi i}^{\pi i} g(z) e^{-k z} d z
$$

Используя уравнение (11) и интегрируя по частям, получим

$$
q_{k}=\frac{k}{2 \pi i} \int_{-\pi i}^{\pi i}\left[e^{h(z)}-h(z)\right] e^{-k z} d z .
$$

Подстановка величин (15) в уравнение (24) приводит к ответу

$$
q_{k}=\frac{(2+a) k}{2 \pi i} \int_{\Gamma_{1}}\left[e^{(2 \lambda+a) / \lambda \cdot w(u) / u}-\frac{2 \lambda+a}{\lambda} \frac{w(u)}{u}\right] u^{(2+a) k-1} d u,
$$

в котором контур $\Gamma_{1}$ начинается в точке $e^{-\pi i /(2+a)}$ и проходит против часовой стрелки вдоль единичной окружности до точки $e^{\pi i /(2+a)}$. Для вычисления $q_{k}$ при $k \gg 1$ заметим, что основной вклад в интеграл приходит из окрестности точки $u=1$, являющейся точкой сингулярности функции $w$. Производя замену переменной интегрирования $u=1+t$, получим

$$
q_{k} \approx \frac{(2+a) k}{2 \pi i} \int_{\Gamma}\left[1+\frac{(2 \lambda+a)^{2}}{2 \lambda^{2}} w^{2}(1+t)\right] e^{(2+a) k t} d t,
$$

где Г - некоторый контур интегрирования справа от начала координат. Теперь можно продолжить $\Gamma$ до $\pm i \infty$ и произвести оценку интеграла при пороговом значении, снизу и сверху от него. 
1. При $\lambda=\lambda_{\mathrm{c}}$, решая уравнение $(23)$, получим

$$
\varphi(1+t)=-\frac{1}{W(-C t)},
$$

где $W(z)-\mathrm{W}$-функция Ламберта, задаваемая уравнением $W(z) e^{W(z)}=z$ с условием $W(0-0)=-\infty$. Подставив формулы $(22)$ и $(26)$ в выражение $(25)$, имеем

$$
q_{k} \approx \frac{(2+a) k}{2 \pi i} \int_{\Gamma}\left\{1+\frac{\left(2 \lambda_{\mathrm{c}}+a\right)^{2} t^{2}}{8 \lambda_{\mathrm{c}}^{2}}\left[1+\frac{2}{W(-C t)}\right]\right\} e^{(2+a) k t} d t .
$$

Поскольку $W(-C t) \approx \ln (C t)$ при $t \rightarrow 0$, а точка $t=0$ есть точка ветвления функции $\ln (C t)$, имеет место соотношение

$$
\begin{aligned}
q_{k} & \approx \frac{(2+a) k}{2 \pi i} \int_{-\epsilon}^{0} \frac{\left(2 \lambda_{\mathrm{c}}+a\right)^{2} t^{2}}{8 \lambda_{\mathrm{c}}^{2}} e^{(2+a) k t}\left(\frac{2}{\ln |C t|-\pi i}-\frac{2}{\ln |C t|+\pi i}\right) d t \approx \\
& \approx \frac{(2+a)\left(2 \lambda_{\mathrm{c}}+a\right)^{2} k}{4 \lambda_{\mathrm{c}}^{2}} \int_{-\epsilon}^{0} \frac{t^{2} e^{(2+a) k t}}{\ln ^{2}|t|} d t .
\end{aligned}
$$

Подстановка $v=-(2+a) k t$ приводит к

$$
q_{k} \approx \frac{\left(2 \lambda_{\mathrm{c}}+a\right)^{2}}{4 \lambda_{\mathrm{c}}^{2}(2+a)^{2}} \frac{1}{k^{2} \ln ^{2} k} \int_{0}^{+\infty} v^{2} e^{-v} d v=\frac{\left(2 \lambda_{\mathrm{c}}+a\right)^{2}}{2 \lambda_{\mathrm{c}}^{2}(2+a)^{2}} \frac{1}{k^{2} \ln ^{2} k} .
$$

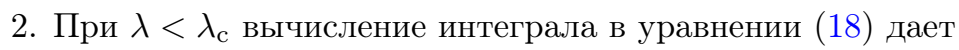

$$
C(u-1)=\left[\frac{1}{2}+\omega-\frac{w(u)}{u-1}\right]^{-1 / 4 \omega-1 / 2}\left[\frac{1}{2}-\omega-\frac{w(u)}{u-1}\right]^{1 / 4 \omega-1 / 2},
$$

где $\tau=w(u) /(u-1)$ и $\omega=\sqrt{1 / 4-\kappa}$. Поэтому при $u \rightarrow 1$ имеем

$$
w(u) \approx(u-1)\left[\frac{1}{2}-\omega+C(u-1)^{\Lambda}\right], \quad \Lambda=\frac{4 \omega}{1-2 \omega} .
$$

Подставив эти соотношения в выражение $(25)$ и вычислив скачок функции $(u-1)^{\Lambda}$ на разрезе, получим

$$
\begin{aligned}
q_{k} & \approx \frac{(2+a) k}{2 \pi i} \int_{\Gamma}\left\{1+\frac{(2 \lambda+a)^{2} t^{2}}{2 \lambda^{2}}\left[\left(\frac{1}{2}-\omega\right)^{2}+C t^{\Lambda}\right]\right\} e^{(2+a) k t} d t \approx \\
& \approx \frac{(2+a) k}{2 \pi i} \int_{-\epsilon}^{0} \frac{(2 \lambda+a)^{2} t^{2}}{2 \lambda^{2}} e^{(2+a) k t} C e^{\Lambda \ln |t|}\left[e^{-i \Lambda \pi}-e^{i \Lambda \pi}\right] d t \approx \\
& \approx-\frac{(2+a)(2 \lambda+a)^{2} k C \sin \Lambda \pi}{2 \lambda^{2} \pi} \int_{-\epsilon}^{0} t^{2} e^{(2+a) k t}|t|^{\Lambda} d t .
\end{aligned}
$$

Подставляя $v=-(2+a) k t$, имеем

$$
q_{k} \approx-\frac{(2 \lambda+a)^{2} C \sin \Lambda \pi}{2 \lambda^{2}(2+a)^{2+\Lambda} \pi} \frac{\Gamma(3+\Lambda)}{k^{2+\Lambda}}=C_{1} \frac{1}{k^{2+\Lambda}} .
$$




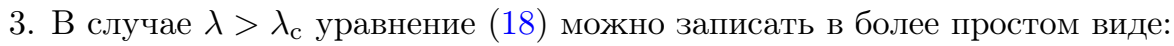

$$
C(u-1)=\left[\left(\frac{w(u)}{u-1}-\frac{1}{2}\right)^{2}+\eta^{2}\right]^{-1 / 2} \exp \left\{-\frac{1}{2 \eta}\left[\frac{\pi}{2}+\operatorname{arctg}\left[\frac{1}{\eta}\left(\frac{w(u)}{u-1}-\frac{1}{2}\right)\right]\right]\right\}
$$

где $\eta=\sqrt{\kappa-1 / 4}$. Применяя асимптотику (20), при $\eta \ll 1$ можно переписать это уравнение как

$$
C(u-1)=\left(\frac{1}{2}-\frac{w(u)}{u-1}\right)^{-1} \exp \left\{-\frac{1}{2 \eta}\left[\pi+\eta\left(\frac{1}{2}-\frac{w(u)}{u-1}\right)^{-1}\right]\right\}
$$

После подстановки $u=1+t$ получаем

$$
w(1+t)=\frac{t}{2}\left\{1+\frac{1}{W\left(C t e^{\pi / 2 \eta}\right)}\right\}
$$

Подставив это равенство в формулу (25), имеем

$$
q_{k} \approx \frac{(2+a) k}{2 \pi i} \int_{\Gamma}\left\{1+\frac{(2 \lambda+a)^{2} t^{2}}{8 \lambda^{2}}\left[1+\frac{2}{W\left(C t e^{\pi / 2 \eta}\right)}\right]\right\} e^{(2+a) k t} d t
$$

В этом выражении только член, содержащий W-функцию Ламберта, сингулярен и приводит к ненулевому вкладу, тем самым

$$
q_{k} \approx \frac{(2+a)(2 \lambda+a)^{2} k}{8 \lambda^{2} \pi i} \int_{\Gamma} \frac{t^{2} e^{(2+a) k t}}{W\left(C t e^{\pi / 2 \eta}\right)} d t .
$$

После замены переменной интегрирования $z=W\left(C t e^{\pi / 2 \eta}\right)$ получим

$$
q_{k} \approx \frac{(2+a)(2 \lambda+a)^{2} k}{8 C^{3} \lambda^{2} \pi i} e^{-3 \pi / 2 \eta} \int_{\Gamma} z(z+1) \exp \left[C^{-1}(2+a) e^{-\pi / 2 \eta} k z e^{z}+3 z\right] d z
$$

Интегрируя в этом выражении по частям, получаем ответ:

$$
\begin{aligned}
q_{k} & \approx \frac{(2 \lambda+a)^{2}}{8 C^{2} \lambda^{2} \pi i} e^{-\pi / \eta} \int_{\Gamma} z e^{2 z} d \exp \left[C^{-1}(2+a) e^{-\pi / 2 \eta} k z e^{z}\right] \approx \\
& \approx-\frac{(2 \lambda+a)^{2}}{8 C^{2} \lambda^{2} \pi i} e^{-\pi / \eta} \int_{\Gamma}(2 z+1) \exp \left[C^{-1}(2+a) e^{-\pi / 2 \eta} k z e^{z}+2 z\right] d z
\end{aligned}
$$

С применением метода седловой точки [22] при $z=-1$ приходим к окончательному выражению

$$
q_{k} \approx \frac{(2 \lambda+a)^{2}}{4 C^{3 / 2}(2+a)^{1 / 2}(2 \pi)^{1 / 2} \lambda^{2}} k^{-1 / 2} \exp \left[-C^{-1}(2+a) e^{-\pi / 2 \eta-1} k-\frac{3 \pi}{4 \eta}-\frac{3}{2}\right] .
$$

Теорема доказана. 


\section{4. ОБСУЖДЕНИЕ}

Чтобы привести модель к достаточно простому виду, допускающему аналитическое решение, мы предположили, что число новых связей, добавляемых на каждом шаге, задается распределением Пуассона. Здесь мы ослабим это предположение и рассмотрим дискретные распределения общего вида. Более точно, будем на каждом шаге добавлять новый узел и с вероятностями $\tau_{k}$ будем добавлять $k$ новых связей, связывающих новый узел со старыми в соответствии с преимущественным правилом соединения, как описано в разделе 2. Оказывается, что при этом большинство результатов настоящей работы остаются справедливыми с некоторыми модификациями. Например, как и в случае перколяционного порога, имеет место следующая теорема.

Теорема 5. Предположим, что существует $t_{0}>0$ такое, что ряд $\sum_{k=0}^{\infty} e^{t k} \tau_{k}$ сходится при $|t|<t_{0}$. Тогда кривая перколяиионного перехода в данной модели задается уравнением

$$
\sigma^{2}=\frac{16 \mu^{4}+32 a \mu^{3}+24 a^{2} \mu^{2}+8 a^{3} \mu+a^{4}}{\left(16 a^{2}+32 a\right) \mu^{2}+\left(16 a^{3}+32 a^{2}\right) \mu+4 a^{4}+8 a^{3}},
$$

где

$$
\mu=\sum_{k} k \tau_{k}, \quad \sigma^{2}=\sum_{k} k^{2} \tau_{k}-\mu^{2}
$$

суть математическое ожидание и дисперсия распределения $\boldsymbol{\tau}$.

ДоКАЗАТЕЛЬСтво тЕОРЕМЫ 5 аналогично доказательству теоремы 1 и опускается для краткости изложения.

В случае распределения Пуассона $\mu=\sigma^{2}=\lambda$, и выражение (32) сводится к (8).

Заметим, что уравнение (32) применимо также и в других моделях, например в моделях, исследованных в работах [18]-[20], [23]. Так, в работе [23] исследован случай $\tau_{0}=1-\delta$ и $\tau_{2}=\delta$ без введения преимущественного присоединения. Применяя соотношение (32) и производя предельный переход $a \rightarrow \infty$, получим критическое значение $\delta_{\mathrm{c}}=1 / 2-\sqrt{3} / 4$, находящееся в соответствии с результатами работы [23].

\section{Список литературы}

[1] A. L. Barabási, R. Alber, Science, 286:5439 (1999), 509-512, arXiv: cond-mat/9910332.

[2] M. E. J. Newman, A. L. Barabasi, D. J. Watts, The Structure and Dynamics of Networks, Princeton Univ. Press, Princeton, 2006.

[3] L. F. Costa, O. N. Oliveira, Jr, G. Travieso, F. A. Rodrigues, P. R. Villas Boas, L. Antiqueira, M. P. Viana, L. E. Correa Rocha, Adv. Phys., 60:3 (2011), 329-412, arXiv: 0711.3199.

[4] A. L. Barabási, R. Albert, H. Jeong, Physica A, 272:1 (1999), 173-187, arXiv: cond-mat/9907068.

[5] P. L. Krapivsky, S. Redner, F. Leyvraz, Phys. Rev. Lett., 85:21 (2000), 4629-4632, arXiv: cond-mat/0005139.

[6] S. N. Dorogovtsev, J.F.F. Mendes, Phys. Rev. E, 63:2 (2001), 025101, 4 pp., arXiv: cond-mat/0009065.

[7] A. Barrat, M. Barthélemy, A. Vespignani, Phys. Rev. Lett., 92:22 (2004), 228701, 4 pp., arXiv: cond-mat/0401057. 
[8] R. Albert, A. L. Barabási, Phys. Rev. Lett., 85:24 (2000), 5234-5237, arXiv: cond-mat/ 0005085.

[9] R. Albert, A.L. Barabási, Rev. Modern Phys., 74:1 (2002), 47-97, arXiv: cond-mat/ 0106096.

[10] R. Cohen, K. Erez, D. Ben-Avraham, S. Havlin, Phys. Rev. Lett., 85:21 (2000), 4626-4628, arXiv: cond-mat/0007048.

[11] D. S. Callaway, M. E. J. Newman, S. H. Strogatz, D. J. Watts, Phys. Rev. Lett., 85:25 (2000), 5468-5471, arXiv: cond-mat/0007300.

[12] S. N. Dorogovtsev, A.V. Goltsev, J.F.F. Mendes, Rev. Modern Phys., 80:4 (2008), 1275-1335, arXiv: 0705.0010.

[13] Чжун-Хао Сюй, Я. Хигучи, Чунь-Хуа Ху, ТМФ, 172:1 (2012), 28-39.

[14] P. Erdős, A. Rényi, Publ. Math., 6 (1959), 290-297.

[15] P. Erdős, A. Rényi, Pub. Math. Inst. Hung. Acad. Sci. Ser. A, 5 (1960), 17-61.

[16] D.S. Callaway, J.E. Hopcroft, J. M. Kleinberg, M. E. J. Newman, S. H. Strogatz, Phys. Rev. E, 64:4 (2001), 041902, 7 pp., arXiv: cond-mat/0104546.

[17] S. N. Dorogovtsev, J.F.F. Mendes, A. N. Samukhin, Phys. Rev. E, 64:6 (2001), 066110, 11 pp., arXiv: cond-mat/0106141.

[18] J. Kim, P. L. Krapivsky, B. Kahng, S. Redner, Phys. Rev. E, 66:5 (2002), 055101, 4 pp., arXiv: cond-mat/0203167.

[19] S. Coulomb, M. Bauer, Eur. Phys. J. B, 35:3 (2003), 377-389, arXiv: cond-mat/0212371.

[20] P. L. Krapivsky, B. Derrida, Physica A, 340:4 (2004), 714-724, arXiv: cond-mat/0408161.

[21] В. Ф. Зайцев, А. Д. Полянин, Справочник по обыкновенным дифференциальным уравнениям, Физматлит, М., 2001.

[22] G. Polya, G. Latta, Complex Variables, John Wiley and Sons, New York-London-Sydney, 1974.

[23] D. Lancaster, J. Phys. A, 35:5 (2002), 1179-1194.

Поступила в редакцию 19.03.2015, после доработки 27.04.2015 